\title{
Parametric characterization of non-uniformly polarized beams
}

\author{
J.M. Movilla, G. Piquero, R. Martínez-Herrero, P.M. Mejías \\ Departamento de Óptica, Facultad de Ciencias Físicas, Universidad Complutense, 28040 Madrid, Spain
}

Received 22 July 1997; revised 6 November 1997; accepted 7 January 1998

\begin{abstract}
A new overall parameter is defined, namely, the generalized degree of polarization, which involves the spatial distribution of the polarization of a light beam. This parameter is introduced on the basis of a general treatment recently proposed [Optics Lett. 22 (1997) 206] to characterize partially polarized fields, which extends the Stokes-Mueller formalism to describe the intensity moments of a quasimonochromatic beam. The main properties and the physical meaning of this parameter with regard to the spatial inhomogeneities of the polarization state are analysed, and a general measurement procedure is proposed. (C) 1998 Elsevier Science B.V.
\end{abstract}

\section{Introduction}

As is well known [1-5], the spatial characterization of light beams by means of overall spatial parameters have been defined in the scalar case. In fact, it was assumed so far that the radiation has uniform polarization properties over its cross-sectional area. For this kind of fields the Stokes parameters provide a well-known tool for a systematic analysis of the state of polarization. In practice, however, a problem arises when radiation exhibits spatial variations in the polarization state, for example, in birefringent Nd:YAG rods [6]. In such cases, the phase difference between the orthogonal components of the electric field vector has no fluctuations (the field behaves as totally polarized at each point), but its polarization state (linear, circular or elliptical) would depend on the position across the aperture (we refer to this kind of fields as non-uniformly totally polarized (NUTP) beams). A general simple way to create NUTP beams is to combine two orthogonally linearly polarized beams, which have tailored intensity and/or phase distributions (see, for example, Refs. [7-9]). This would generate spatial inhomogeneities in the polarization across the beam profile. The relative importance of these inhomogeneities would be closely related to the intensity values over the region where they appear. Consequently, it would be interesting to describe and characterize these features by means of some kind of overall parameter, understood as a measure of the uniformity of the polarization state of the field over those crosssectional regions where the beam intensity is significant. This is the aim of this paper.

To this end, some kind of link should be established between the spatially distributed state of polarization and the second-order intensity moments of the beam (in particular, the beam width and the far-field divergence). In this connection, the Stokes-Mueller formalism has been quite recently extended [10] to describe the intensity moments of partially non-uniformly polarized quasimonochromatic beams. On the basis of this generalized formalism, we will introduce in the next section a new overall parameter, namely, the so-called generalized degree of polarization, $\mathbf{P}$, which characterizes the spatial distribution of the polarization over the beam profile. This is of particular relevance concerning the international standards that evaluate the polarization status of a general beam [11]. Its physical meaning is illustrated by means of an example in Section 3 , and a general experimental procedure that can be used to measure $\mathbf{P}$ is explained in Section 4. Finally, Section 5 summarizes the main conclusions of this paper.

\section{Formalism and definitions}

We will consider quasimonochromatic optical fields represented as stationary random processes, propagating as 
a beam (paraxial approach) along, say, the $z$-axis. It has been shown [12] that, to be consistent with the Maxwell equations, the transverse nature of the electromagnetic field demands a nonzero transverse cross-polarization component in addition to the longitudinal component of the field vector along the beam axis. It was shown for Gaussian beams that the longitudinal component as well as the cross-polarization component are weaker than the principal polarization component by 1 and 2 orders of magnitude in $\left(k_{0} w_{0}\right)^{-2}$, where $k_{0}$ is the mean wavenumber and $w_{0}$ the waist size of the beam [12]. Taking this into account, we will assume in the following that, for the beams we handle in the present work, the product $\left(k_{0} w_{0}\right)^{-1}$ takes small enough values to ignore the effects of both kinds of components. Note, for example, that, in the visible range, $k_{0} \sim 10^{4} \mathrm{~mm}^{-1}$ and $w_{0}>10^{-2} \mathrm{~mm}$, so that $\left(k_{0} w_{0}\right)^{-2}<$ $10^{-4}$, and the above assumption is fulfilled.

Accordingly, from now on, we denote the associated dimensionless field vector perpendicular to the $z$-axis by

$\boldsymbol{E}(\boldsymbol{r}, z ; \omega)=\left(E_{s}(\boldsymbol{r}, z ; \omega), E_{p}(\boldsymbol{r}, z ; \omega)\right)$,

where $\omega$ represents the angular frequency and $s$ and $p$ are the axes of an arbitrary orthogonal coordinate system. In Eq. (1), $E_{s}$ and $E_{p}$ are the field components along these directions (we use in this paper $s$ and $p$, not $x$ and $y$, to describe the polarization direction of the field). For convenience, the spatial variables $(x, y)=\boldsymbol{r}$ are considered here dimensionless variables. They are defined as the product of $k_{0}$ by the Cartesian coordinates of the point at which we are evaluating the field.

Let us first introduce the electric field cross-spectral density matrix [13,14], $G$, which, for our purposes, we write in the form

$G\left(\boldsymbol{r}_{1}, \boldsymbol{r}_{2}, z ; \omega\right)=\overline{\boldsymbol{E}^{+}\left(\boldsymbol{r}_{1}, z ; \omega\right) \boldsymbol{E}\left(\boldsymbol{r}_{2}, z ; \omega\right)}$,

where the overbar symbolizes an ensemble average (the field can, in general, be partially polarized) and

$\boldsymbol{E}^{+}=\left(\begin{array}{l}E_{s}^{*} \\ E_{p}^{*}\end{array}\right)$

is the adjoint of the field vector $\boldsymbol{E}$ (see Eq. (1)). In terms of the matrix $G$, the so-called Wigner matrix, $H$, can be defined as follows $[10,15]$,

$H(\boldsymbol{r}, \boldsymbol{\eta}, z)=\frac{1}{k_{0}^{2}} \int G\left(\boldsymbol{r}+\frac{\boldsymbol{s}}{2}, \boldsymbol{r}-\frac{\boldsymbol{s}}{2}, z\right) \exp (i \boldsymbol{s} \cdot \boldsymbol{\eta}) \mathrm{d} \boldsymbol{s}$,

where $\boldsymbol{\eta}=(u, v)$, with $u$ and $v$ representing angles of propagation (without taking the evanescent waves into account). For simplicity, the dependence on $\omega$ has been omitted. To write Eq. (4) it has been implicitly considered that the optical devices are non-dispersive systems for the assumed beam spectral widths. If we write

$\boldsymbol{R} \equiv(x, y, u, v)=(\boldsymbol{r}, \boldsymbol{\eta})$, the Stokes matrices associated to a partially polarized field are defined in the form [10]

$S_{i}=\iint \boldsymbol{R}^{\mathrm{t}} \boldsymbol{R} \operatorname{tr}\left(\sigma_{i} H(\boldsymbol{r}, \boldsymbol{\eta})\right) \mathrm{d} \boldsymbol{r} \mathrm{d} \boldsymbol{\eta}, \quad i=0,1,2,3$,

where the superscript ' $t$ ' indicates the transposed matrix, the symbol 'tr' denotes the trace of the matrix, and

$\sigma_{0}=\left(\begin{array}{ll}1 & 0 \\ 0 & 1\end{array}\right), \quad \sigma_{1}=\left(\begin{array}{cc}1 & 0 \\ 0 & -1\end{array}\right)$,

$\sigma_{2}=\left(\begin{array}{ll}0 & 1 \\ 1 & 0\end{array}\right), \quad \sigma_{3}=\left(\begin{array}{cc}0 & i \\ -i & 0\end{array}\right)$.

Note that $\sigma_{1}, \sigma_{2}$ and $\sigma_{3}$ are the Pauli matrices. In addition, matrix $S_{0}$ involves all (measurable) second-order intensity moments of the beam. In fact, the matrix $S_{0}$ can be written as follows

$S_{0}=\left(\begin{array}{cc}W^{2} & \psi \\ \psi^{\mathrm{t}} & \Phi^{2}\end{array}\right)$

where

$W^{2}=\left(\begin{array}{ll}{\left[x^{2}\right]} & {[x y]} \\ {[x y]} & {\left[y^{2}\right]}\end{array}\right)$,

$\Psi=\left(\begin{array}{ll}{[x u]} & {[x v]} \\ {[y u]} & {[y v]}\end{array}\right)$,

$\Phi^{2}=\left(\begin{array}{ll}{\left[u^{2}\right]} & {[u v]} \\ {[u v]} & {\left[v^{2}\right]}\end{array}\right)$,

and the square brackets [ ] represent spatial averages, namely

$[\alpha \beta]=\int \alpha \beta \operatorname{tr} H \mathrm{~d} \boldsymbol{r} \mathrm{d} \boldsymbol{\eta}=\left([\alpha \beta]_{s s}+[\alpha \beta]_{p p}\right)$,

$\alpha, \beta=x, y, u, v$,

where

$[\alpha \beta]_{i j}=\int \alpha \beta H_{i j} \mathrm{~d} \boldsymbol{r} \mathrm{d} \boldsymbol{\eta}, \quad i, j=s, p$.

It should be noted that the averages $\left[r^{2}\right]$ and $\left[\eta^{2}\right]$ are related with the usual second-order intensity moments by means of the following equations:

$\left\langle r^{2}\right\rangle=\frac{\left[r^{2}\right]}{4 \pi^{2} k_{0}^{2} I}$,
$\left\langle\eta^{2}\right\rangle=\frac{\left[\eta^{2}\right]}{4 \pi^{2} I}$,

where $\left\langle r^{2}\right\rangle$ (squared length) and $\left\langle\eta^{2}\right\rangle$ (dimensionless quantity) represent the standard (squared) beam width and divergence, respectively (see, for example, Ref. [4]), and I is the total power of the beam (integrated throughout the beam profile).

Within the framework of the above formalism, let us now introduce a new parameter, $\mathbf{P}$, in terms of the traces of the Stokes matrices

$\mathbf{P}=\left[\frac{\left(\operatorname{tr} S_{1}\right)^{2}+\left(\operatorname{tr} S_{2}\right)^{2}+\left(\operatorname{tr} S_{3}\right)^{2}}{\left(\operatorname{tr} S_{0}\right)^{2}}\right]^{1 / 2}$. 
We call $\mathbf{P}$ the generalized degree of polarization of the beam because it formally resembles the well-known expression

$P=\left[\frac{s_{1}^{2}+s_{2}^{2}+s_{3}^{2}}{s_{0}^{2}}\right]^{1 / 2}$,

where $P$ is the usual degree of polarization [16], and $s_{i}$, $i=0,1,2,3$, are the standard Stokes parameters. It can be shown [10] that, for a general partially polarized beam, we have

$\left(\operatorname{tr} S_{0}\right)^{2} \geq\left(\operatorname{tr} S_{1}\right)^{2}+\left(\operatorname{tr} S_{2}\right)^{2}+\left(\operatorname{tr} S_{3}\right)^{2}$,

which, in a sense, is similar to the expression [16]

$s_{0}^{2} \geq s_{1}^{2}+s_{2}^{2}+s_{3}^{2}$.

Taking this into account, it follows at once that

$0 \leq \mathbf{P} \leq 1$.

In addition, the case $\mathbf{P}=1$ corresponds to totally polarized beam $s$ whose polarization state is independent of the transversal coordinate $\boldsymbol{r}$. In other words, $\mathbf{P}=1$ means uniformly totally polarized fields. On the other hand, when the field components $E_{p}$ and $E_{s}$ are mutually incoherent (natural light) $\mathbf{P}=0$. Note however, that $\mathbf{P}=0$ does not necessarily imply completely unpolarized beams in the usual sense, i.e., natural light: it suffices to consider, for example, beams with random spatial (but not temporal) variations in the state of polarization across their apertures. It is also important to note that, without any prior knowledge, no conclusion can be inferred from a particular value of $\mathbf{P}(\neq 1)$ concerning whether the field is partially polarized or exhibits a spatial dependence of the polarization state over the beam profile (NUTP beams).

In addition, it can be shown that the parameter $\mathbf{P}$ is invariant under rotation of the Cartesian coordinate axes. This result follows from a well-known theorem of matrix algebra, namely, the invariance of the traces under rotation.

To know how $\mathbf{P}$ propagates through optical systems it suffices to recall the corresponding propagation laws of the Stokes matrices, namely [10]

$S_{n}^{\prime}=M S_{n} M^{\mathrm{t}}, \quad n=0,1,2,3$,

and

$S_{n}^{\prime}=\sum_{m=0}^{3} L_{n m} S_{m}, \quad n, m=0,1,2,3$,

where $S_{n}^{\prime}$ and $S_{m}^{\prime}$ denote the Stokes matrices at the output and input plane, respectively, $M$ represents the $4 \times 4$ $A B C D$ matrix of a non-polarizing first-order system, and $L_{n m}$ are the elements of the Mueller matrix that characterizes a polarizing optical system (it is assumed that each $L_{n m}$ is independent of $\boldsymbol{r}$ ). Accordingly, application of Eqs.
(19) and (20) provides $\mathbf{P}$ at the output plane in terms of the values of the Stokes matrices at the initial plane. In particular, $\mathbf{P}$ does not change under propagation through any $4 \times 4 A B C D$ optical system in the following two cases: (i) uniform totally polarized field $(\mathbf{P}=1)$; (ii) beams for which $S_{1}=S_{2}=S_{3}=0$. Natural light belongs to this second type of fields.

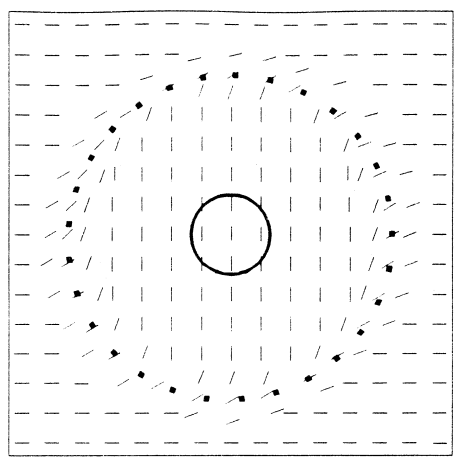

a)

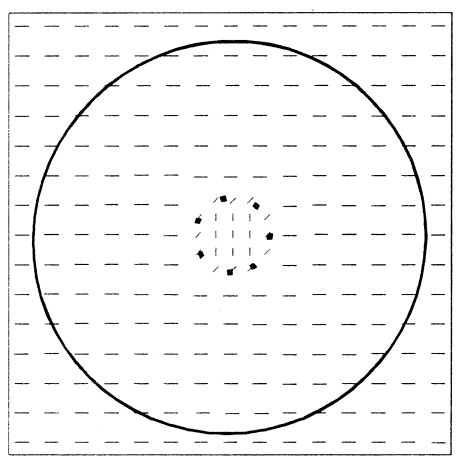

b)

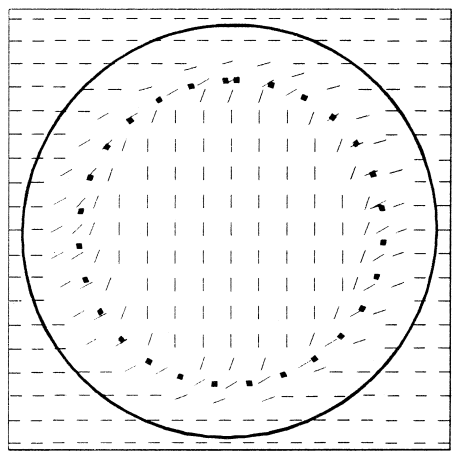

c)

Fig. 1. Schematic diagrams of the spatial distribution of the polarization of a beam whose electric field amplitude is given by Eq. (21). In all the figures the radii of the dotted and solid-line circles are $a^{-1}$ and $w_{0}$, respectively. Each mark indicates the azimuth of the linear polarization state at each point across the beam profile. 


\section{Physical meaning of parameter $P$ for NUTP beams}

To get deeper insight into the physical meaning of parameter $\mathbf{P}$, let us now consider, for illustrative purposes, a NUTP beam whose electrical field is represented at plane $z=0$ by the following Jones vector

$$
\begin{aligned}
\boldsymbol{E}(r)= & E_{0}\left(\cos \left\{\frac{\pi}{2} \exp \left[-(a r)^{16}\right]\right\},\right. \\
& \left.\sin \left\{\frac{\pi}{2} \exp \left[-(a r)^{16}\right]\right\}\right) \exp \left[-\left(\frac{r}{w_{0}}\right)^{2}\right],
\end{aligned}
$$

where $E_{0}$ is an amplitude factor, $r$ represents here the radial polar coordinate, and $w_{0}$ and $a$ are constants. It is easy to see from Eq. (21) that the global intensity of this beam, $\left|E_{s}\right|^{2}+\left|E_{p}\right|^{2}$, is purely Gaussian, and, at each point of its cross-section area, the field is linearly polarized whose azimuth depends on the radial distance to the beam centre.

Figs. 1a-1c show the spatially distributed polarization of the beam for several values of the product $a w_{0}$. Inspection of these figures reveals that inside a circle whose radius is $\approx a^{-1}$ the beam approximately behaves as a uniformly linearly polarized field with negligible $s$-component, whereas outside such a circle, the beam remains uniformly polarized but now with $E_{p} \approx 0$.

The abrupt transition between both regimes follows from the super-Gaussian structure of the exponential factor within the cosine and sine functions. Also, in Fig. 1a $\left(a w_{0} \ll 1\right)$ the field behaves as $p$-polarized over that cross-sectional area over which the beam intensity takes appreciable values. In fact the beam intensity outside the dotted circle is negligible. In Fig. $1 \mathrm{~b}\left(a w_{0} \gg 1\right)$, the beam behaves as $s$-polarized over the entire beam profile except over a small area around its centre. Finally, in Fig. 1c $\left(a w_{0} \approx 1\right)$ the global beam intensities associated to the $s$ and $p$-polarized regions are balanced.

Let us now plot the value of $\mathbf{P}$ in terms of the product $a w_{0}$ (see Fig. 2). We get $\mathbf{P} \approx 1$ for the cases sketched in

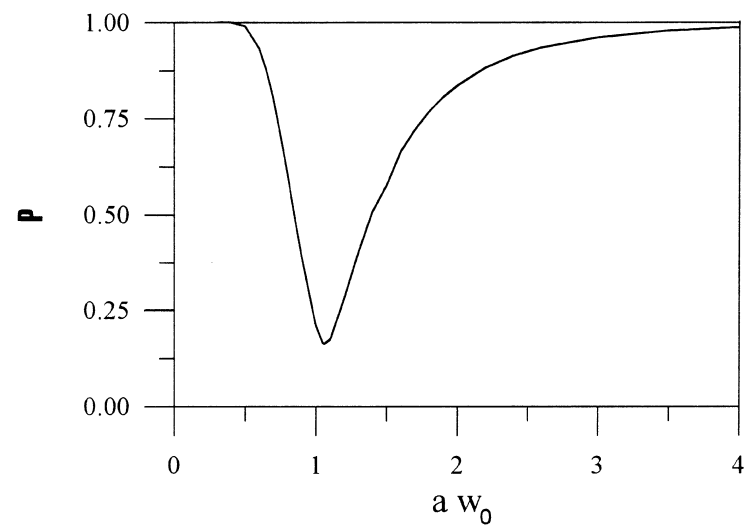

Fig. 2. Generalized degree of polarization $\mathbf{P}$ versus the product $a w_{0}$ for the example represented by Eq. (21).
Figs. 1a and 1c, but $\mathbf{P}$ drastically reduces $(\mathbf{P} \leq 0.3)$ when $a w_{0}$ approaches 1 . Taking this into account, it can be said that, for NUTP beams, the parameter $\mathbf{P}$ is a measure of the uniformity of the polarization state of the beam over those cross-sectional regions where the beam intensity is important. In other words, values of $\mathbf{P}$ close to 1 means that the beam has uniform polarization at least over the beam profile region where the intensity takes the most significant values and the value $\mathbf{P} \approx 0$ means that no overall definite state of polarization can be assigned over such peak intensity region.

\section{Measurement of $P$}

Parameter $\mathbf{P}$ can be measured in different ways. We will next propose a simple experimental procedure that resembles the well-known method applied to determine the standard Stokes parameters. By using a polarizer and a quarter-wave plate, one only needs to measure the global intensity and the second-order beam moments (beam width and divergence) for several orientations of the transmission axis of the polarizer, and solve the corresponding relations obtained from the propagation law (20). The method follows four steps:

1. We measure the total power $I$, the (squared) beam width and the (squared) divergence of the beam at the observation plane in the free propagation case.

2. We measure the same parameters as before but now the beam travels through a polarizer which accepts linear polarization in the azimuth $\alpha=0^{\circ}$, where shows $\alpha$ the orientation of the transmission axis of the polarizer with respect to the $s$-axis.

3. The same measurements as in step 2 but now with $\alpha=45^{\circ}$.

4. We again measure the same parameters as above but now the beam propagates successively through the quarter-wave plate (whose fast axis makes an angle $0^{\circ}$ with the $s$-axis) and the polarizer oriented so as to transmit the component in the azimuth $\alpha=45^{\circ}$.

Of course, the observation plane remains unchanged during the procedure. After application of Eq. (20) the resulting equations for the traces of the matrices $S_{i}$ (in terms of the averages defined in Eq. (10)) are

$$
\begin{aligned}
\operatorname{tr} S_{0}= & {\left[r^{2}\right]_{\mathrm{FP}}+\left[\eta^{2}\right]_{\mathrm{FP}}, } \\
\operatorname{tr} S_{1}= & \frac{1}{T_{1}-T_{2}}\left\{\left[2\left(\left[r^{2}\right]_{\alpha=0^{\circ}}+\left[\eta^{2}\right]_{\alpha=0^{\circ}}\right)\right]\right. \\
& \left.-\left(T_{1}+T_{2}\right) \operatorname{tr} S_{0}\right\}, \\
\operatorname{tr} S_{2}= & \frac{1}{T_{1}-T_{2}}\left\{\left[2\left(\left[r^{2}\right]_{\alpha=45^{\circ}}+\left[\eta^{2}\right]_{\alpha=45^{\circ}}\right)\right]\right. \\
& \left.-\left(T_{1}+T_{2}\right) \operatorname{tr} S_{0}\right\},
\end{aligned}
$$




$$
\begin{aligned}
\operatorname{tr} S_{3}= & \frac{1}{T_{1}-T_{2}}\left\{2\left[\left(\left[r^{2}\right]_{\lambda / 4, \alpha=45^{\circ}}\right)+\left(\left[\eta^{2}\right]_{\lambda / 4, \alpha=45^{\circ}}\right)\right]\right. \\
& \left.-\left[\left(T_{1}+T_{2}\right) \operatorname{tr} S_{0}\right]\right\},
\end{aligned}
$$

where the subscript 'FP' denotes free propagation to the observation plane and the subscript ' $\lambda / 4, \theta=45^{\circ}$ ' refers to step 4 of our procedure. Remember that $\left[r^{2}\right]$ and $\left[\eta^{2}\right]$ are connected with the respective standard measurable second-order intensity moments through Eqs. (12) and (13). We have also included in Eqs. (22) the coefficients $T_{1}$ and $T_{2}$ that characterize the major and minor principal transmittances of any imperfect polarizer (see, for example, Refs. [17] and [18]). From the above expressions, the value of $\mathbf{P}$ can be inferred at once.

\section{Conclusions}

On the basis of the Stokes-Mueller formalism, the distributed state of polarization of a non-uniformly totally polarized quasimonochromatic light beam has been characterized by means of a new overall parameter, namely, the so-called generalized degree of polarization, $\mathbf{P}$. The limit values of $\mathbf{P}$ have been given, along with some invariance properties. Moreover, $\mathbf{P}$ is shown to be closely related with the well-known second-order intensity moments, such as the beam width and the far-field divergence. This enables us to measure $\mathbf{P}$ by means of a simple experimental procedure that resembles the method used to determine the standard Stokes parameters.

\section{Acknowledgements}

The research work leading to this paper has been supported by the Comisión Interministerial de Ciencia y Tecnología of Spain, under project TAP96-2333-E, within the framework of EU-1269 project. We also thank an anonymous referee for attaching a copy of the relevant pages of Ref. [15], for the authors.

\section{References}

[1] S. Lavi, R. Prochaska, E. Keren, Appl. Optics 27 (1988) 3696.

[2] M.J. Baastians, Optik 82 (1989) 173.

[3] A.E. Siegman, Proc. SPIE 1224 (1990) 2.

[4] J. Serna, R. Martínez-Herrero, P.M. Mejías, J. Opt. Soc. Am. A 8 (1991) 1096.

[5] H. Weber, Opt. Quantum Electron. 24 (1992) 1027.

[6] Q. Lü, S. Dong, H. Weber, Opt. Quantum Electron. 27 (1995) 777.

[7] S.C. Tidwell, D.H. Ford, W.D. Kimura, Appl. Optics 29 (1990) 2234.

[8] S.C. Tidwell, G.H. Kim, W.D. Kimura, Appl. Optics 32 (1993) 5222.

[9] J.M. Movilla, G. Piquero, R. Martinez-Herrero, P.M. Mejias, On the spatial characterization of polarized light beams, in: A. Giesen, M. Morin (Eds.), Proc. 4th International Workshop on Laser Beam and Optics Characterization, VDI-Technologiezentrum, in press.

[10] R. Martínez-Herrero, P.M. Mejías, J.M. Movilla, Optics Lett. 22 (1997) 206.

[11] Draft International Standard ISO/DIS 12005, Test methods for laser beam parameters: Polarization.

[12] S. Simon, E.C.G. Sudarshan, N. Mukunda, Appl. Optics 26 (1987) 1589.

[13] L. Mandel, E. Wolf, Optical Coherence and Quantum Optics, chap. 6, Cambridge University Press, Cambridge, 1995.

[14] E. Wolf, Phys. Rev. D 13 (1976) 869.

[15] D.S. Bugnolo, H. Bremmer, The Wigner distribution matrix for the electric field in a stochastic dielectric with computer simulation, in: P.W. Hawkes (Ed.), Advances in Electronics and Electron Physics, Academic Press, New York, 1983, pp. 299-389.

[16] M. Born, E. Wolf, Principles of Optics, ch. X, Pergamon Press, Oxford, 1993.

[17] J.M. Bennett, H.E. Bennett, Polarization, in: W.E. Driscoll, W. Vaughan (Eds.), Handbook of Optics, Section 10, McGraw-Hill, New York, 1978.

[18] J. Krása, J. Jiřička, M. Lokajíček, Phys. Rev. E 48 (1993) 3184. 